\title{
Density functional approach to helium at finite temperature
}

\author{
Thierry Biben ${ }^{1}$ and Daan Frenkel \\ FOM-Institute for Atomic and Molecular Physics (AMOLF), Kruislaan 407, \\ 1098 SJ Amsterdam, The Netherlands \\ Received 11 April 2002 \\ Published 27 September 2002 \\ Online at stacks.iop.org/JPhysCM/14/9077
}

\begin{abstract}
We report an application of the general formalism of density functional theory (DFT) for quantum fluids at finite temperature to the case of helium. Using this approach, we compute the liquid-vapour coexistence curve and the surface tension of helium at low temperatures. We observe that the range of the interface is much larger than the usual 10-90 surface thickness, and we find that the DFT reproduces the $T^{7 / 3}$ temperature dependence of the surface tension. This implies that capillary-wave effects are, at least partially, accounted for by the density functional.
\end{abstract}

The aim of density functional theory (DFT) is to construct a functional relation between the free energy of a system of interacting particles and its single-particle density profile. It should be stressed that, in spite of the central role played by the single-particle density, many-body effects are in principle fully incorporated in DFT. In fact, the general formalism is based on an exact mapping between the fluid of interacting quantum particles and an effective fluid of independent particles. This independent-particle reference fluid has the same single-particle density as the interacting many-body system. However, the wavefunctions of this effective fluid are not related to the many-body wavefunctions of the interacting fluid. In fact, DFT provides no information about the true wavefunction of the system. Although DFT has been applied mainly to the study of interacting many-electron systems, the formalism is completely general and applies to any type of quantum fluid, either bosonic or fermionic. Of course, the specific form of the density functional will depend on the nature of the system studied.

Once the density functional is known, it becomes possible to compute the equilibrium properties of an inhomogeneous system, such as the density profile of an interface and surface tension. However, the surface of a fluid in equilibrium is, itself, subject to fluctuations and it is not obvious that such capillary-wave fluctuations are properly included in DFT. In fact, this point has been a matter of debate for many years. Much of this discussion has centred

1 Permanent address: Groupe de Recherche sur les Phénomènes hors Équilibre, Laboratoire de Spectrométrie Physique, Université Joseph Fourier, CNRS UMR5588, BP87 F-38402 Saint Martin d'Hères Cedex, France. 
on classical fluids. Evans showed that the square-gradient approach is able to reproduce a capillary-wave-like behaviour in a self-consistent way [1]. However he later concluded [2] that this inclusion of capillary-wave-like modes cannot account for roughening, so the squaregradient approximation still misses some contributions. Evans concludes that it is probably difficult to build approximations based on DFT that lead to a correct treatment of capillary waves, and that it is necessary to mix DFT treatments with other capillary-wave models, as done by Ebner and Saam [3].

In 1975, Ebner and Saam [3] proposed a density functional to describe the helium liquidvapour interface. The Ebner-Saam functional was based on an integral equation proposed by Mihara and Puff [4]. Using this approach Ebner and Saam found an extremely low value for the surface tension. This led them to conclude that their density functional failed to account properly for the zero-point contribution of capillary waves. Subsequently, Ebner and Saam [5] were able to obtain a very good estimate of the surface tension, using an elegant renormalization procedure, based on a quantum hydro-dynamical study of the liquid-vapour interface. This led them to conclude that capillary waves are the main contribution to the surface energy of helium. However, the recent density functionals introduced by Stringari and Treiner [6] and Dupont-Roc et al [7] (the so-called Paris-Orsay functional) lead to excellent values for the surface tension, without introducing explicitly the zero-point contribution of capillary waves. This would suggest that, on the contrary, capillary waves do not contribute significantly to the surface tension.

It is then difficult to conclude from a density functional study whether capillary waves are taken into account or not, and in what proportion. An interesting result obtained by Saam [5] is that the surface tension should decrease as $T^{7 / 3}$ when the temperature increases. This effect can be attributed unambiguously to the capillary waves. In this paper we show that a simple extension of the Paris-Orsay functional to finite temperatures leads to precisely such a behaviour. This indicates that the Paris-Orsay functional does take the effect of capillary waves into account, at least qualitatively. If this is indeed the case, then there is no contradiction between the conclusion by Ebner and Saam about the zero-point contribution of capillary waves to the surface tension and the results obtained with the DFT of [7]. The present study is divided into three parts. The first part is dedicated to a brief presentation of the general formalism at finite temperature [8]; this formalism has been successfully applied recently to the study of fermions $[9,10]$. In the second part we investigate the particular case of a homogeneous fluid; we argue that the exchange-correlation grand potential is effectively constant at low temperature. Using the finite-temperature DFT we study the phase diagram of helium. The third part deals with inhomogeneous fluids and more precisely with the liquid-vapour interface. In this part, we focus on the temperature dependence of the surface tension.

\section{Density functional theory at finite temperature}

In 1965 Mermin showed [8] that it is possible to define a functional of the one-particle density $\Omega[n]=F[n]-\mu \int n(\vec{r}) \mathrm{d} \vec{r}$, whose minimum corresponds to the equilibrium density profile of the fluid. This functional is an extension of the functional developed by Hohenberg and Kohn [11] for the particular case $T=0 \mathrm{~K}$. Mermin's free-energy functional takes the form

$$
F[n]=\operatorname{Tr}\left\{\rho[n]\left(K+V_{\text {int }}+V_{\text {ext }}+\frac{1}{\beta} \ln \rho[n]\right)\right\}
$$

where $\rho[n]$ is the density matrix functional of an interacting fluid, at inverse temperature $\beta=1 / k_{B} T$, having the density profile $n(\vec{r})$. $K$ represents the kinetic energy operator, $V_{\text {int }}$ is the inter-particle potential and $V_{\text {ext }}$ is the external potential. This free-energy functional can 
be rewritten

$$
\begin{gathered}
F[n]=\operatorname{Tr}\left\{\rho[n]\left(K+\frac{1}{\beta} \ln \rho[n]\right)\right\}+\frac{1}{2} \int n(\vec{r}) n\left(\vec{r}^{\prime}\right) g\left(\vec{r}, \vec{r}^{\prime}\right) V_{\text {int }}\left(\vec{r}, \vec{r}^{\prime}\right) \mathrm{d} \vec{r} \mathrm{~d} \vec{r}^{\prime} \\
+\int n(\vec{r}) V_{\text {ext }}(\vec{r}) \mathrm{d} \vec{r}
\end{gathered}
$$

where $g\left(\vec{r}, \vec{r}^{\prime}\right)$ is the pair distribution function of the inhomogeneous fluid.

For a weakly coupled system $\left(\beta V_{\text {int }} \ll 1\right)$ the density matrix functional can be approximated by the density matrix functional $\rho^{0}[n]$ of a non-interacting system, and at large enough distances the pair distribution function tends to unity. It is usual to extract these two contributions from the functional, writing

$F[n]=\mathcal{F}^{0}[n]+\frac{1}{2} \int n(\vec{r}) n\left(\vec{r}^{\prime}\right) V_{\text {int }}\left(\vec{r}, \vec{r}^{\prime}\right) \mathrm{d} \vec{r} \mathrm{~d} \vec{r}^{\prime}+\int n(\vec{r}) V_{\text {ext }}(\vec{r}) \mathrm{d} \vec{r}+\Omega^{x c}[n]$,

where

$$
\mathcal{F}^{0}[n]=\operatorname{Tr}\left\{\rho^{0}[n]\left(K+\frac{1}{\beta} \ln \rho^{0}[n]\right)\right\}
$$

and $\Omega^{x c}$ is the so-called exchange-correlation grand potential, defined by relation (3). Expression (3) corresponds to a generalization of the Kohn-Sham procedure at zero temperature [12]. It is important to notice that $\mathcal{F}^{0}[n]$ is an intrinsic functional that depends neither on $V_{\text {int }}$ nor on $V_{\text {ext }}$. Obviously $\Omega^{x c}[n] \equiv 0$ for a non-interacting fluid. The minimization of (3) in the grand canonical ensemble leads to

$$
\mu=\frac{\delta \mathcal{F}^{0}}{\delta n(\vec{r})}+\int n\left(\vec{r}^{\prime}\right) V_{\text {int }}\left(\vec{r}, \vec{r}^{\prime}\right) \mathrm{d} \vec{r}^{\prime}+V_{\text {ext }}(\vec{r})+\frac{\delta \Omega^{x c}}{\delta n(\vec{r})}
$$

for interacting fluids. In the absence of interaction, we have

$$
\mu=\frac{\delta \mathcal{F}^{0}}{\delta n(\vec{r})}+V_{\text {ext }}(\vec{r})
$$

A comparison of the last two relations shows that solving equation (5) is formally equivalent to the solution of a problem of non-interacting particles placed in an effective potential:

$$
\mathcal{V}^{e f f}=\int n\left(\vec{r}^{\prime}\right) V_{i n t}\left(\vec{r}, \vec{r}^{\prime}\right) \mathrm{d} \vec{r}^{\prime}+V_{\text {ext }}(\vec{r})+\frac{\delta \Omega^{x c}}{\delta n(\vec{r})},
$$

where $n(\vec{r})$ here represents the equilibrium density profile. We then have to solve selfconsistently the one-particle Schrödinger equation

$$
\left(-\frac{\hbar^{2}}{2 m} \Delta+\mathcal{V}^{e f f}[n(\vec{r})]\right) \phi_{\nu}=\epsilon_{\nu} \phi_{\nu}
$$

together with the condition that

$$
n(\vec{r})=\sum_{\nu} f_{\nu} \phi_{\nu}^{*}(\vec{r}) \phi_{\nu}(\vec{r})
$$

where $f_{v}$ is the occupation number of level $v$. This can be done in the following way. From an initial guess of $n(\vec{r})$, one can calculate the effective potential using (7), and the wavefunctions of the non-interacting fluid placed in this external potential solving (8). As a result we obtain a new estimate of the density profile using (9). The equilibrium density profile is obtained by iterating this procedure, together with a prescription to mix input and output profiles, in order to obtain a convergence. 
Once again, the equivalence between the problem of interacting particles and the effective problem of non-interacting particles only applies to $n(\vec{r})$. The wavefunctions $\phi_{v}(\vec{r})$ have no physical meaning.

At equilibrium, the density matrix of the non-interacting system is

$$
\rho^{o}=\frac{\exp \left[-\beta\left(K+\mathcal{V}^{e f f}-\mu N\right)\right]}{\Xi}
$$

with

$$
\Xi=\operatorname{Tr}\left\{\exp \left[-\beta\left(K+\mathcal{V}^{e f f}-\mu N\right)\right]\right\} .
$$

Introducing these two relations into (4) leads to the following expression for $\mathcal{F}^{o}(n)$ :

$$
\mathcal{F}^{o}=-\frac{1}{\beta} \ln \Xi+\int\left(\mu-\mathcal{V}^{e f f}[n(\vec{r})]\right) n(\vec{r}) \mathrm{d} \vec{r} .
$$

The procedure we describe here is a straightforward generalization of the Hohenberg-KohnSham procedure to zero temperature. At $T=0, F[n]$ reduces to the Hohenberg and Kohn functional [11] and $\mathcal{F}^{0}[n]$ to the Kohn-Sham intrinsic kinetic-energy functional [12], so $\Omega^{x c}[n]$ goes to $E^{x c}[n]$, the exchange-correlation energy functional.

\section{The homogeneous fluid: phase diagram}

For a homogeneous fluid of density $n, \mathcal{V}^{e f f}(n)$ is simply a constant, so the solution of equation (8) with periodic boundary conditions in a cubic box of volume $V$ is straightforward. From (12) and (3), one can easily check that in the thermodynamic limit the expression for the free-energy density of a Bose fluid at equilibrium is

$$
\frac{F(n)}{V}=\frac{1}{\beta} \frac{4}{\sqrt{\pi}} \frac{1}{\Lambda^{3}} \int_{0}^{+\infty} x^{2} \log \left[1-z_{0} \exp \left(-x^{2}\right)\right] \mathrm{d} x+\frac{1}{2} n^{2} \int V_{i n t}(r) \mathrm{d} \vec{r}+\frac{\Omega_{h}^{x c}}{V}+\mu_{0} n,
$$

where we have introduced the notations

$$
\begin{aligned}
& \mu_{0}=\mu-\mathcal{V}^{e f f}(n) \\
& z_{0}=\exp \left(\beta \mu_{0}\right)
\end{aligned},
$$

$\Lambda=\sqrt{h^{2} / 2 \pi m k_{B} T}$ is the de Broglie thermal wavelength and $\Omega_{h}^{x c}$ is the exchange-correlation grand potential for a homogeneous fluid.

The density of the fluid is related to the effective fugacity $z_{0}$ through the usual relation:

$$
n=\frac{4}{\sqrt{\pi}} \frac{1}{\Lambda^{3}} \int_{0}^{+\infty} \frac{x^{2}}{-1+\frac{1}{z_{0}} \exp x^{2}} \mathrm{~d} x \quad \text { if } n<n_{c} \equiv \frac{4}{\sqrt{\pi}} \frac{1}{\Lambda^{3}} \int_{0}^{+\infty} \frac{x^{2}}{-1+\exp x^{2}} \mathrm{~d} x
$$

$n=n_{0}+n_{c}$ otherwise

where $n_{0}$ is the number of particles in the ground state for the effective non-interacting system. $n_{c}$ is the critical density for the Bose condensation of the effective non-interacting fluid.

Differentiating relation (13) with respect to temperature, at fixed density, we obtain, after some algebra, an expression for the entropy:

$$
\frac{S}{V}=-\frac{5}{2} \frac{1}{T} \frac{1}{\beta \Lambda^{3}} \frac{4}{\sqrt{\pi}} \int_{0}^{+\infty} x^{2} \log \left[1-z_{0} \exp \left(-x^{2}\right)\right] \mathrm{d} x-\frac{\partial \Omega_{h}^{x c} / V}{\partial T}-\frac{\mu_{0}}{T} n .
$$

The first term is the ideal Bose gas contribution.

When $T$ goes to zero, the critical density goes to zero. This property implies that for a fixed density, it is possible to find a finite temperature for which there is a Bose condensation. Below that temperature, $\mu_{0}$ is zero and $z_{0}=1$, so expression (16) reduces to

$$
\frac{S}{V}=-\frac{5}{2} \frac{1}{T} \frac{1}{\beta \Lambda^{3}} \frac{4}{\sqrt{\pi}} \int_{0}^{+\infty} x^{2} \log \left[1-\exp \left(-x^{2}\right)\right] \mathrm{d} x-\frac{\partial \Omega_{h}^{x c} / V}{\partial T} .
$$




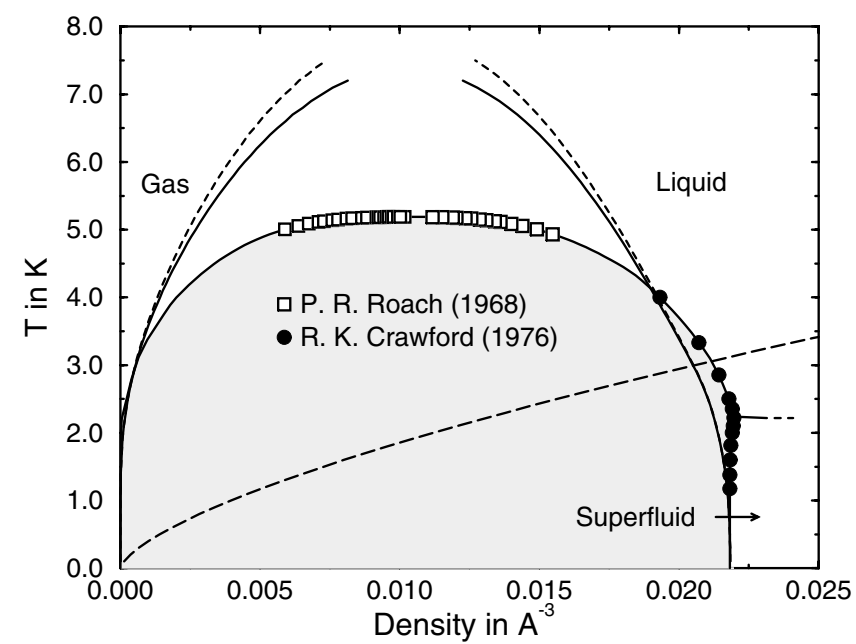

Figure 1. Phase diagram: DFT versus experimental results (from [18]and [19]). The grey area is the experimental liquid (superfluid)-vapour coexistence region. The two solid curves are the results of the extension of the Paris-Orsay functional, and the two dashed curves represent the extension of the functional suggested by Dalfovo et al. Long dashes represent the Bose condensation line of the effective non-interacting fluid.

As a result, at $T=0 \mathrm{~K}$,

$$
\frac{S}{V}=-\frac{\partial \Omega_{h}^{x c} / V}{\partial T}
$$

From this last expression we conclude that $\partial \Omega_{h}^{x c}(n) / \partial T$ must vanish at $T=0 \mathrm{~K}$.

It is important to notice that the derivative appearing in (18) has been calculated at fixed density. However, as was pointed out by Perrot and Dharma-wardana [13], in the grand canonical ensemble we must consider the derivative at fixed chemical potential.

The fact that $\partial \Omega_{h}^{x c}(n) / \partial T$ vanishes at $T=0 \mathrm{~K}$ simply proves that, to a first approximation, the explicit dependence in temperature of $\Omega^{x c}$ can be neglected at low temperature. We must now discuss the implicit dependence due to the variation of the bulk density when temperature goes to zero at fixed chemical potential. This is complicated by the fact that it is necessary to know the asymptotic behaviour of the effective fugacity, which in turn requires the knowledge of the asymptotic behaviour of the effective potential at fixed chemical potential. We show in the appendix that, if the behaviour of the exchange-correlation grand potential is 'regular enough', we obtain that $\partial n /\left.\partial T\right|_{\mu}=0$ at $T=0 \mathrm{~K}$. As the simplest approximations satisfy this 'regularity criterion', we shall neglect the temperature dependence of the exchange-correlation grand potential, approximating $\Omega^{x c}[n]$ by its expression at $T=0 \mathrm{~K}, E^{x c}[n]$. A comparison of the DFT phase diagram with experimental data shows that below $1 \mathrm{~K}$ this approximation is reasonable (figure 1).

An explicit expression for the free energy can be obtained using

$$
\left\{\begin{array} { l } 
{ \int V _ { \text { int } } ( r ) \mathrm { d } \vec { r } = b } \\
{ \frac { E _ { h } ^ { x c } ( n ) } { N } = \frac { c } { 2 } n ^ { 1 + \gamma } }
\end{array} \quad \text { with } \left\{\begin{array}{l}
b=-888.81 \mathrm{~K}^{3} \\
c=1.04554 \times 10^{7} \mathrm{~K}^{3(1+\gamma)} \\
\gamma=2.8
\end{array}\right.\right.
$$


for the Paris-Orsay functional, and

$$
\left\{\begin{array} { l } 
{ \int V _ { i n t } ( r ) \mathrm { d } \vec { r } = b ^ { \prime } } \\
{ \frac { E _ { h } ^ { x c } ( n ) } { N } = \frac { c _ { 2 } } { 2 } n ^ { 2 } + \frac { c _ { 3 } } { 3 } n ^ { 3 } }
\end{array} \quad \text { with } \left\{\begin{array}{l}
b^{\prime}=-718.99 \mathrm{~K} \AA^{3} \\
c 2=-2.411857 \times 10^{4} \mathrm{~K} \AA^{6} \\
c 3=1.858496 \times 10^{6} \mathrm{~K} \AA^{9}
\end{array}\right.\right.
$$

for the functional suggested by Dalfovo et al [14]. One can easily compute the phase diagram from the free-energy expression (13), by performing a double-tangent construction. The phase diagrams obtained with both functionals are shown in figure 1, together with experimental data.

The most obvious difference between DFT and experimental results is the critical behaviour of the liquid-vapour transition. The overestimation of the liquid-vapour critical point is not surprising since both approximations (19) and (20) are of the mean-field type. The estimated DFT critical point is $7.3 \mathrm{~K}$. Although this value is not particularly close to the experimental value $T_{c}=5.19 \mathrm{~K}$, it is an improvement over classical studies of the LennardJones (LJ) fluid. The classical approach led to an estimated critical temperature $T_{c} \simeq 13.5 \mathrm{~K}$. Quantum corrections have been estimated using a Wigner expansion in powers of $\hbar$ for the particular case of neon [15], with the result that the critical temperature is reduced by nearly $4 \%$. However quantum effects are much stronger for helium (the critical temperature is only $38 \%$ of the classical estimate). Hence, for helium, the Wigner expansion is expected to converge poorly, if at all. In contrast, the simple quantum mean-field functional presented in this paper gives an estimate of the critical temperature that is closer to experimental data than either the classical or semi-classical estimates. Of course, the mean-field approximation leads to a parabolic behaviour close to the critical point. It is possible to improve the critical behaviour by keeping the pair distribution function that appears in expression (2). However, this improvement makes it necessary to have reliable integral equations to estimate the pair distribution function at any density and temperature.

The second difference between experimental data and DFT is the Bose condensation. In DFT, the Bose condensation is due to the ideal term. As a consequence the transition occurs at too high a value of temperature, and again the critical behaviour is not correct. However, most DFTs are not constructed to lead to a good description of critical behaviour-close to a critical point, long-ranged many-particle correlations play a key role. In DFT, these correlations are usually approximated in a very crude way, or ignored altogether. In contrast, outside the critical region, DFT is a powerful tool to describe the density of coexisting phases and the interface. This is the reason why we focus on the behaviour of the coexisting densities rather than the critical behaviour. This leads us to the final and possibly most troublesome difference: the incorrect behaviour of the bulk liquid density at low temperature. We can see in figure 1 that the density decreases with temperature instead of increasing. This contraction of the liquid phase as temperature increases indicates clearly that the simple prescription used to estimate the exchange-correlation contribution to the functional cannot be completely correct at low temperature.

We conclude from this study that the DFT presented in this paper can be considered as a good zero-order approximation, since it gives a reasonable description of the phase diagram (the right phases at nearly the right place). Improvements of this functional are possible, but require a careful investigation of exchange-correlational effects. 


\section{Inhomogeneous systems}

Following [7], we choose the interaction potential as follows:

$$
V_{\text {int }}(r)= \begin{cases}4 \epsilon\left[\left(\frac{\alpha}{r}\right)^{12}-\left(\frac{\alpha}{r}\right)^{6}\right] & \text { if } r \geqslant h \\ V_{\text {int }}(h)\left(\frac{r}{h}\right)^{4} & \text { otherwise }\end{cases}
$$

with $\epsilon=10.22 \mathrm{~K}, \alpha=2.556 \AA$ and $h=2.377 \AA$ is adjusted so that $\int V_{i n t}(r) \mathrm{d} \vec{r}=b$, as already mentioned in relation (19). The approximation $\Omega^{x c}[n] \equiv E^{x c}[n]$ reads

$$
\Omega^{x c}[n]=\frac{c}{2} \int \bar{n}(\vec{r})^{1+\gamma} n(\vec{r}) \mathrm{d} \vec{r}
$$

where $\bar{n}(\vec{r})$ is a coarse-grained density, obtained by averaging $n(\vec{r})$ in a sphere of radius $h$. The values of $b, c$ and $\gamma$ are given by relation (19).

One can compute the density profile of the fluid in an external potential by minimizing the free energy (3). For a free planar liquid-vapour interface, the external potential is zero and the problem reduces to a one-dimensional one, since all integrals over the transverse coordinates can be calculated analytically. The boundary conditions in the longitudinal $z$ direction are periodic, with the result that there are two liquid-vapour interfaces in the box. The box size $L$ is chosen so that these two interfaces do not interact. We shall return to this point later. Some density profiles are presented in figure 2 (top graph) for temperatures varying in the range 0-2 K. From these data, we can easily compute the structure factor of the interface, using the relation [16]

$$
S(q)=\frac{1}{n_{\infty}^{e}} \int_{-\infty}^{+\infty} \frac{\mathrm{d} n^{e}(z)}{\mathrm{d} z} \exp (\mathrm{i} q z) \mathrm{d} z
$$

where $n_{\infty}^{e}$ is the electron density of the bulk liquid phase. Assuming that the electron density is proportional to the atomic density of helium, we can replace $n^{e}(z)$ by $n(z)$ and compute $|S(q)|^{2}$. This last quantity plays a key role in x-ray reflectivity experiments. The predicted structure factors for the liquid-vapour interface are shown in figure 3 , for temperatures in the range $0-2 \mathrm{~K}$. We can observe the broadening of the interface as temperature increases. At present, the only x-ray scattering data on the structure of the helium liquid-vapour interface were acquired from a relatively thin film of helium adsorbed on a silicon substrate [16]. These experiments cannot be compared directly with the present numerical results. Hopefully, future experiments will be able to probe the structure of thick ('semi-infinite') helium films.

Once the equilibrium profile is obtained, the surface tension can be computed by using the relation

$$
\sigma=\frac{1}{2} \int_{0}^{L}\left(\frac{\Omega[n(z)]}{V}+P\right) \mathrm{d} z
$$

where $P$ is the bulk pressure of the coexisting phases. The bulk pressure can be determined from the expression of the grand potential for a homogeneous system using as input the liquid density measured far from the interface, or from the vapour density. The last choice is in fact the best, since the pressure depends strongly on the density in the liquid phase.

For a classical fluid, the grand potential functional is usually expressed as an integral of a local 'density' $f$ :

$$
\Omega[n(r)]=\int f(n(\vec{r})) \mathrm{d} \vec{r} .
$$




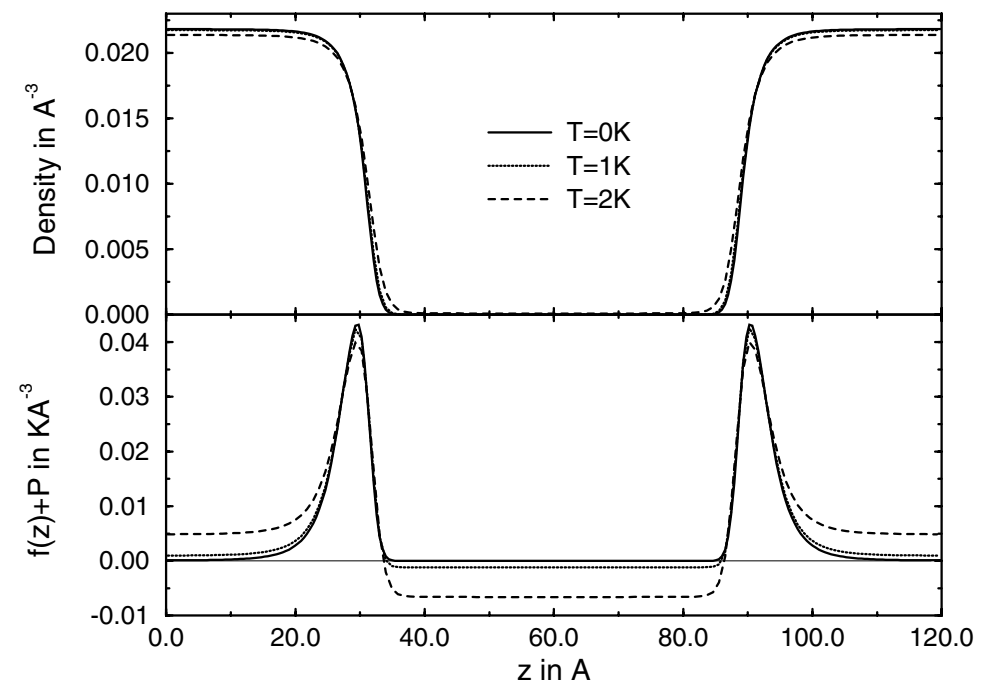

Figure 2. Density profile as a function of temperature, and the corresponding variations of $f(z)+P$. The density profile does not change much between $T=0$ and $1 \mathrm{~K}$. We can also see that the bulk liquid density tends to decrease with temperature instead of increasing.

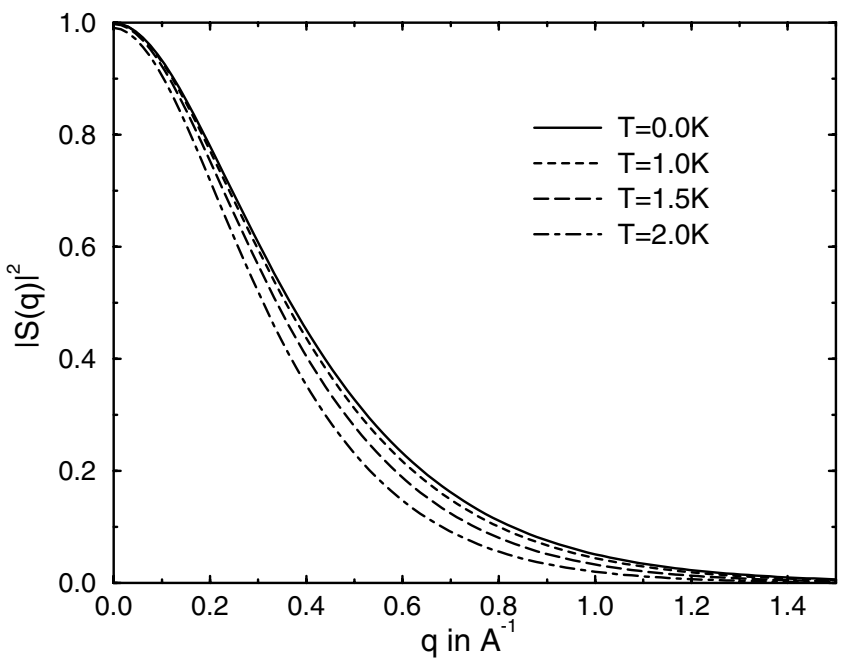

Figure 3. Structure factor of the liquid-vapour interface for a semi-infinite film of helium.

The grand potential density $f(n(\vec{r}))$ has the property that far from the interface $f$ is equal to $-P$, the bulk pressure. This last property is useful since it gives a simple criterion to determine the range of the interface: the range of $f(n(z))+P$. However for a quantum fluid this is not the case at finite temperature since the ideal term contribution is global, and has no natural local expression. To resolve this problem, we artificially spread this global contribution homogeneously over all the volume of the system in relation (24). As a result, the surface tension can still be written in the form

$$
\sigma=\frac{1}{2} \int_{0}^{L}(f(n(z))+P) \mathrm{d} z .
$$


The price we have to pay is that at non-zero temperatures $f(n(z))+P$ does not tend to zero far from the interface, as can be seen in figure 2. The values far from the interface depend on the phase and the box length. If the box size goes to infinity the values tend to zero. At $T=0$ the ideal term vanishes so that $f(n(z))$ is the natural local expression of the grand potential.

As we cannot use the vanishing of $f(n(z))+P$, at finite temperature, to identify the edges of the interface, we have adjusted the box length by looking at the variations of $f(z)+P$ for $T=0 \mathrm{~K}$. We assume that the range of the interface does not vary much at low, but finite, temperature.

It is important to notice that the range of the interface, as defined above, differs from the traditional definition of the 10-90 surface thickness, defined as the distance between the points for which the density is respectively 10 and $90 \%$ of the bulk liquid density. Although the 10-90 surface thickness remains of the order of $6 \AA$, the range of the interface is more than $20 \AA$, with the result that the thickness of a film adsorbed on a substrate should be larger than, say, $30 \AA$ to make sure that the layering of the fluid close to the substrate does not interfere with the interface. For a free-standing film, such as studied in the present paper, the thickness should be larger than $40 \AA$, otherwise the interaction between the two interfaces would lower the value of the surface tension, as can be seen in figure 4 . This figure shows the variation of the computed surface tension with temperature. For a box length $L=120 \AA$ the film thickness is $60 \AA$ and the interfaces are independent, as can be checked in figure 2 for $T=0 \mathrm{~K}$, but for $L=60$ Åthe two interfaces are interacting and the computed surface tension is reduced. A comparison between the computed values and a $T^{7 / 3}$ power law leads to a remarkably good agreement. The $T^{7 / 3}$ law is supposed to be valid at low temperatures, and it is argued in [17] that this scaling should apply at temperatures of the order of $1 \mathrm{~K}$. In figure 5 the temperature dependence of the surface tension is shown in a log-log plot. Even when interfaces are interacting, the $T^{7 / 3}$ law is obeyed very well indeed. The accuracy in the computation of the surface tension is of the order of $0.1 \%$, due to discretization. The value of $\sigma_{0} \equiv \sigma(T=0)$ used for the logarithmic plot has been adjusted to correct the drift due to numerical accuracy that appears when using the DFT value of $\sigma_{0}$, as shown in figure 5. In every case, the adjusted value differs by $<0.04 \%$ from the DFT-computed one. As a result, the simple extension of the Paris-Orsay functional at finite temperature reproduces very well the scaling law:

$$
\sigma(T)=\sigma(T=0)-a T^{7 / 3} .
$$

The measured pre-factor $a=11.52 \times 10^{-3} \mathrm{dyn} \mathrm{cm}^{-1} \mathrm{~K}^{-7 / 3}$ is about a factor of two larger than the value given by Cheng et al [17], $6.5 \times 10^{-3} \mathrm{dyn} \mathrm{cm}^{-1} \mathrm{~K}^{-7 / 3}$, but its order of magnitude is correct. We believe that the $T^{7 / 3}$-behaviour of the functional results from the fact that some capillary-wave-like modes are accounted for by the ideal term. This term is the exact expression of the free energy of a fluid of non-interacting particles confined by the effective potential at temperature $T$. The fluctuations of the resulting 'ideal interface' are then adequately taken into account. There is however an important qualitative difference between the 'ideal interface' and the real one. The localization of the 'ideal interface' is due to the confining effective potential, but the localization of the real interface is due to a spontaneous symmetry breaking process. Therefore Goldstone modes of the real interface should exist that are not taken into account by the ideal term.

\section{Conclusion}

We have presented a simple extension of the zero-temperature density functionals for ${ }^{4} \mathrm{He}$. This extension allows us to obtain a reasonable estimate for both the phase diagram and the 


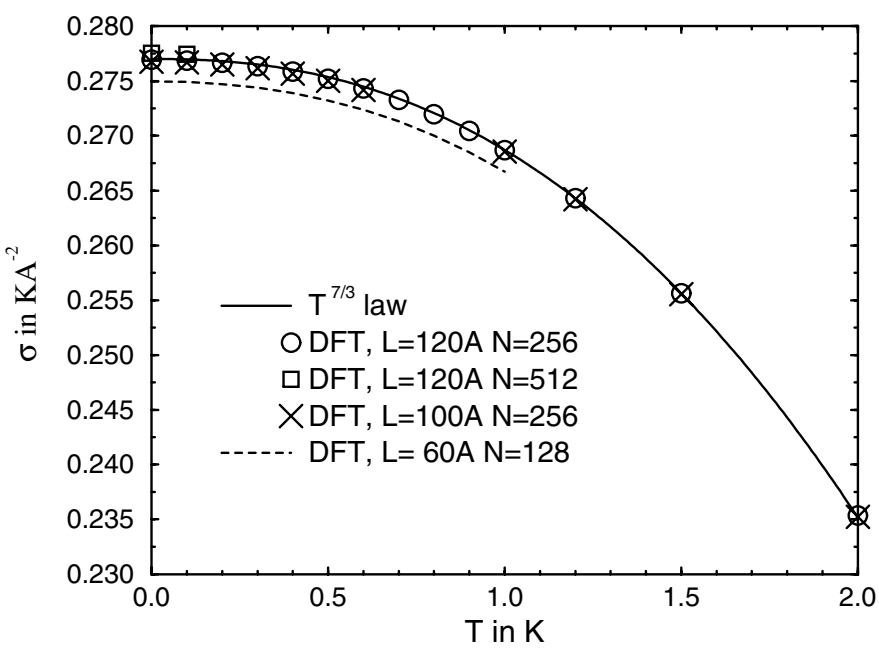

Figure 4. Surface tension as a function of temperature for various box lengths $(L)$. $N$ represents the number of grid points used to perform the resolution of the Schrödinger equation along the $z$ axis. It is important to notice that for $L=60 \AA$, although they are separated by $30 \AA$, the two interfaces are interacting. As a result, the computed surface tension is too small.

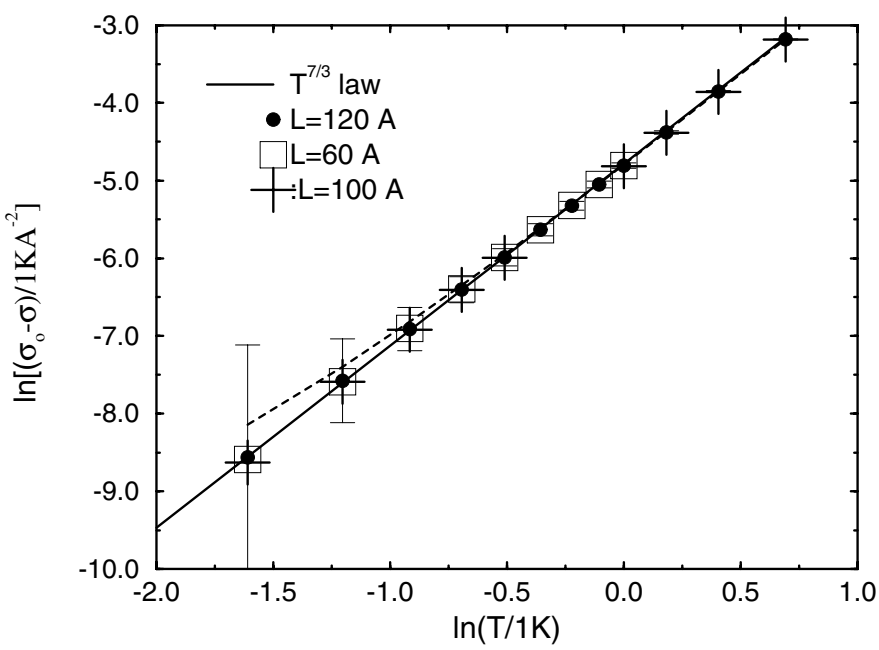

Figure 5. Logarithmic plot of the surface tension for various box lengths. The value of $\sigma_{0} \equiv \sigma(T=0)$ has been corrected as mentioned in the text. We present the uncorrected result (dashed curve) for $L=120 \AA$, and we can see the drift due to the lack of accuracy on the determination of $\sigma(T=0)$. The error-bars correspond to an accuracy of $0.1 \%$ in the computation of the surface tension.

behaviour of the surface tension at low temperatures. The density functional approach that we use is not appropriate in the vicinity of the liquid-vapour critical point. However, far away from $T_{c}$, it leads to realistic density profiles. One remaining problem is that, at low temperatures, the bulk liquid density decreases rather than increases with temperature. We expect that this problem can be solved by properly accounting for the difference in exchange contributions for a system in its ground state and in excited states. The mean-field-like behaviour close to the 
liquid-vapour critical point could be improved by introducing the pair distribution function. However, in both cases the improvement requires a careful study of the exchange-correlation term. We are, at present, investigating possible improvements.

\section{Acknowledgments}

This paper is dedicated to Professor J P Hansen. The work of the FOM Institute is part of the scientific program of FOM and is supported by the Nederlandse Organisatie voor Wetenschappelijk Onderzoek (NWO). Computer time on the Cray C98/4256 at SARA was made available by the Stichting Nationale Computer Faciliteiten (NCF).

\section{Appendix}

\section{A.1 Density behaviour at fixed chemical potential}

We consider only the values of the chemical potential for which the density goes to a finite value when $T \rightarrow 0$, which are the values of physical interest. In this case, there is a finite temperature below which the ideal effective fluid is Bose condensed. This implies that at low temperature, the density is obtained by inverting:

$$
\mu=\mathcal{V}^{e f f}(n)=n \int V_{i n t}(r) \mathrm{d} \vec{r}+\frac{\delta \Omega^{x c}}{\delta n(\vec{r})}(n(\vec{r}) \equiv n) .
$$

Differentiating this expression with respect to temperature, at fixed chemical potential, one obtains

$0=\left.\left\{\int V_{i n t}(r) \mathrm{d} \vec{r}+\frac{\partial}{\partial n}\left[\frac{\delta \Omega^{x c}}{\delta n(\vec{r})}(n(\vec{r}) \equiv n)\right]\right\} \frac{\partial n}{\partial T}\right|_{\mu}+\left.\frac{\partial}{\partial T}\left[\frac{\delta \Omega^{x c}}{\delta n(\vec{r})}(n(\vec{r}) \equiv n)\right]\right|_{n}$.

Now, if we assume that the exchange-correlation grand potential functional is regular enough that

$$
\frac{\delta \Omega^{x c}}{\delta n(\vec{r})}(n(\vec{r}) \equiv n)=\frac{\partial \Omega_{h}^{x c} / V}{\partial n}
$$

then

$$
\left.\frac{\partial}{\partial T}\left[\frac{\delta \Omega^{x c}}{\delta n(\vec{r})}(n(\vec{r}) \equiv n)\right]\right|_{n}=\frac{\partial}{\partial n} \frac{\partial \Omega_{h}^{x c} / V}{\partial T}
$$

is zero at $T=0 \mathrm{~K}$, since at that temperature $\partial\left(\Omega_{h}^{x c} / V\right) / \partial T$ vanishes for all values of the density. As a result, expression (29) is satisfied at $T=0 \mathrm{~K}$ if $\partial n /\left.\partial T\right|_{\mu}=0$ (there is no reason why the pre-factor $\partial \mathcal{V}^{e f f}(n) /\left.\partial n\right|_{T}$ should vanish at $\left.T=0 \mathrm{~K}\right)$.

It is important to notice that relation (30) is satisfied by many approximations for the exchange-correlation grand potential, such as local approximations, non-local approximations including a convolution kernel and square-gradient corrections.

\section{References}

[1] Evans R 1981 Mol. Phys. 421169

[2] Evans R 1990 Les Houches 1988 Liquids at Interfaces ed J Charvolin, J F Joanny and J Zinn-Justin (Amsterdam: North-Holland)

[3] Ebner C and Saam W F 1975 Phys. Rev. B 12923

[4] Mihara N and Puff R D 1968 Phys. Rev. 174221

[5] Saam W F 1975 Phys. Rev. B 12163

[6] Stringari S 1987 and Treiner J 1987 Phys. Rev. B 368369 
[7] Dupont-Roc J, Himbert M, Pavloff N and Treiner J 1990 J. Low Temp. Phys. 8131

[8] Mermin N D 1965 Phys. Rev. A 1371441

[9] Alavi A and Frenkel D 1992 J. Chem. Phys. 979249

[10] Alavi A, Kohanoff J, Parrinello M and Frenkel D 1994 Phys. Rev. Lett. 732599

[11] Hohenberg P and Kohn W 1964 Phys. Rev. B 136864

[12] Kohn W and Sham L J 1965 Phys. Rev. A 1401133

[13] Perrot F and Dharma-wardana M W C 1984 Phys. Rev. A 302619

[14] Dalfovo F, Lastri A, Pricaupenko L, Stringari S and Treiner J 1995 Phys. Rev. B 521193

[15] Hansen J P and Weis J J 1969 Phys. Rev. 188151

[16] Lurio L B, Rabedeau T A, Pershan P S, Silvera I F, Deutsch M, Kosowsky S D and Ocko B M 1992 Phys. Rev. Lett. 682628

[17] Cheng E, Cole M W, Saam W F and Treiner J 1992 Phys. Rev. B 4613967

[18] Roach P R 1968 Phys. Rev. 170213

[19] Crawford R K 1976 Rare Gas Solids vol 1, ed M L Klein and J A Venables (New York: Academic) p 663 